\title{
TTR
}

Traduction, terminologie, re?daction

\section{Chantal Bouchard. La langue et le nombril : histoire d'une obsession québécoise. Montréal, coll. Nouvelles études québécoises, Fides, 1998, 303 p.}

\section{Houria Daoud-Brikci}

Volume 11, numéro 2, 2e semestre 1998

Psychanalyse et traduction : voies de traverse

Psychoanalysis and Translation: Passages Between and Beyond

URI : https://id.erudit.org/iderudit/037343ar

DOI : https://doi.org/10.7202/037343ar

Aller au sommaire du numéro

Éditeur(s)

Association canadienne de traductologie

ISSN

0835-8443 (imprimé)

1708-2188 (numérique)

Découvrir la revue

Citer ce compte rendu

Daoud-Brikci, H. (1998). Compte rendu de [Chantal Bouchard. La langue et le nombril : histoire d'une obsession québécoise. Montréal, coll. Nouvelles études québécoises, Fides, 1998, 303 p.] TTR, 11(2), 243-247.

https://doi.org/10.7202/037343ar d'utilisation que vous pouvez consulter en ligne.

https://apropos.erudit.org/fr/usagers/politique-dutilisation/ 


\section{COMPTES RENDUS}

Chantal Bouchard. La langue et le nombril ; histoire d'une obsession québécoise. Montréal, coll. Nouvelles études québécoises, Fides, 1998, $303 \mathrm{p}$.

La langue et le nombril de Chantal Bouchard vient à propos jeter une lumière inédite sur le débat houleux relatif à la langue parlée au Québec qui, après trente ans de nationalisme linguistique, est demeuré contradictoire, le fait français en Amérique du Nord reposant sur un frêle équilibre linguistique, social, économique et politique. Il complète en quelque sorte ce qui peut être qualifié de cycle de publications* dans ce domaine, ces quatre dernières années. En quoi La langue et le nombril marque-t-il un toumant positif et prometteur dans les études sur la langue au Québec?

Tout d'abord, il ne s'inscrit pas, loin s'en faut, dans le courant victimisant ou culpabilisant que nous avons connu jusqu'ici. Ensuite, il ne repose ni sur une démonstration instrumentaliste (politique ou autre) ni sur des enquêtes informelles à partir d'échantillons de sous-groupes de la société québécoise, et dont le résultat est souvent révélateur d'un discours métalinguistique subjectif et alarmiste, criant pour conjurer hic et nunc, ici et maintenant, maintenant ou jamais, un consensus linguistique, identitaire et culturel, comme si une langue, quelle qu'elle soit, pouvait évoluer sur commande, en un tour de baguette magique, au mépris des contraintes historiques prescrites par l'espace et le temps.

Pour notre auteure, ce consensus est en voie de construction, tout comme la langue est en voie de normalisation, normalisation souvent occultée par ignorance du phénomène scientifique même de l'évolution des langues humaines, eu égard aux pressions historiques intérieures et extérieures qu'il est inutile de rappeler ici. En outre, le propos de l'auteure dépasse les étapes historiques, qu'elle passe en revue, une à une, synchroniquement et diachroniquement, sous forme de plusieurs tableaux synoptiques des changements historiques qui ont infléchi la trajectoire de l'évolution du français au Québec, décrivant le combat pour la spécificité française et la volonté inébranlable de protéger le fait français. Aujourd'hui, ce qu'il convient de retenir de ces étapes, ce sont les 
marqueurs géographiques, politiques et sociologiques de l'évolution de la langue au Québec, y compris le stade qu'elle a atteint aujourd'hui, stade que la littérature larmoyante sur l'état de la langue, dans l'aveuglement qui la caractérise, ignore superbement.

Pour se démarqquer des plaidoyers défaitistes présentés abusivement au public sous le label d'" essais * sur la langue par des auteurs qui ne connaissent pas le fonctionnement des langues, l'auteure a choisi la voie originale de livrer au lecteur le débat le plus public qui soit, et le plus passionnant qui puisse se lire non sur l'histoire de la langue des Québécois, mais sur les relations qu'ils ont entretenues avec leur langue par les médias interposés, la presse écrite essentiellement. Le lecteur est tenu en haleine, non tant par le style du récit historique linéaire et chronologique, sobre et rigoureux, soucieux du détail et scrupuleux, mais surtout par le climat psychologique agité qui caractérise ces relations quasiment ombilicales. Nous assistons alors au développement d'un discours sur la langue qualifié par d'autres auteurs de " maniaques ", en tout cas bouillonnant, tendu, contradictoire, jamais consensuel, souvent caricatural, parfois sombre, toujours viscéral, culpabilisateur et alarmiste. Le fait même de détailler cette relation intime à la langue depuis les dossiers, rapports, chroniques, articles, décrets, lois et autres interventions des temps les plus reculés de l'histoire du Québec (1760 et surtout après 1867) jusqu'au jour d'aujourd'hui, revient en fait à une tentative d'exorciser une psychose subtilement créée par les pouvoirs sournois de la colonisation, puis savamment entretenue et distillée à doses meurtrières par les constructions déroutantes et peu sécurisantes d'une image de soi dégradée (communes, d'ailleurs, à tout peuple minoritaire doublement exproprié de son territoire et de sa langue).

Qui culpabilise qui au sujet de la langue, objet et sujet d'obsession narcissique chez les Québécois - parler de sa langue revient a parler de soi, dit l'auteure - ? Tout a commencé par l'expression assassine French Canadian Patois, dont l'auteure a cru nécessaire de rappeler qu'elle n'était, étymologiquement parlant, nullement péjorative, mais qui a fait boule de neige dans l'affect des Canadiens français de l'époque. Mais aussi, cette insécurité linguistique et identitaire s'est trouvée confortée par une élite québécoise (d'autrefois et d'aujourd'hui, phénomène néocolonial vécu par la plupart des pays anciennement colonisés, et magistralement décrit par Frantz Fanon, l'écrivain psychiatre 
algérien d'origine antiliaise, tout comme il est dénoncé par le linguiste Louis Calvet), désabusée, elle-même insécurisée. Il n'y a qu'à voir, pour s'en convaincre, les diverses appellations qui ont prévalu dans les divers discours sur la langue, dans une tentative désespérée de trouver une définition heureuse du plus petit au plus grand dénominateur commun à la langue et à l'appartenance géostratégique : joual, franco-québécois, français parisien, français de France, français canadien, français québécois, français du Québec, français nord-américain, français approximatif, français standard, français international, français de qualité, français tout court, français de la francophonie, français universel, français mythique, etc., montrant à l'évidence une identité qui se cherche dans une constante redéfinition. Cette élite fait semblant de ne pas voir la remarquable et inexorable évolution collective de la langue au Québec, évolution indissociable des transformations sociales, économiques et politiques du pays. En d'autres termes, il y a lieu, laisse entendre l'auteure, de cesser d'évaluer la langue au Québec à l'aune du nombril, pour aller chercher dans le discours scientifique et universitaire, lorsqu'il est lucide et objectif, les clés de toute problématique linguistique, fut-elle de par sa complexité particulière au Québec, dans une dynamique collective (et non dans celle des sous-groupes, comme le souligne l'auteure), mais aussi globale, au niveau des mutations que connaissent la plupart des langues du monde, y compris celles dont le parcours n'a pas été entravé, et qui sont bien rares. On découvre alors dans une telle démarche la québécitude sereine d'une langue française partie résolument à la conquête de sa propre norme, ne se définissant plus ailleurs mais sur son propre territoire, s'adaptant à sa situation * aux confins du bilinguisme et de la géographie * et admettant courageusement qu'avec la France, le lien filial est devenu lointain et somme toute secondaire.

Ce livre en dit vraiment plus que son titre. Mais si l'on s'en tenait stricto sensu à celui-ci, on comprendrait pourquoi, selon l'auteure, le nombrilisme des Québécois était représenté par le catholicisme, la natalité et l'agriculture, l'exclusion volontaire de la vie politique et économique du pays pendant près de deux siècles, supprimant en même temps tout apport français ou francophone de l'extérieur du Canada. Le nombrilisme, c'est également le repli sur soi et sur son territoire qui fait surgir des images de soi construites par soi sur soi et celles renvoyées, quand elles ne sont pas instituées, par l'Autre. Bref, le nombrilisme fait partie de l'idéologie de la survivance et relève des peurs ancestrales des Québécois, 
lesquelles se sont fort heureusement transformées dès les années 70 en. forces positives : le moi haîssable est peu à peu devenu aimable, le Québec s'étant réapproprié une partie du gâteau économique qui lui revenait, souvent au monde extérieur et participant au concert des nations. A l'intérieur comme à l'extérieur du pays, des centres d'études québécoises et des congrès locaux et internationaux inscrivent à leur ordre du jour des études sur l'évolution du français au Québec. Le Québécois a finalement réussi à jeté les oripeaux du nombrilisme étriqué dans lequel il s'était enfermé, dirions-nous, en position fotale, pour se lancer dans une bataille constructive : couper coate que coute l'isolement, attirer l'attention du monde sur son existence, contrer l'anglicisation systématique des communautés immigrées et séduire celles-ci par une politique linguistique valorisante, à la mesure des nouvelles ambitions du Québec.

Les histoires de nombril sont souvent des histoires d'amour. Dans celle-ci, l'amour fou des Québécois pour la langue française ne s'est pas démenti une seule fois au fil des pages : passion de la reconstruction historique des canadianismes, survalorisation, quoique conjoncturelle - bien qu'elle ait toujours, et bien naturellement, ses adeptes - des parlers populaires, chasse obsessionnelle aux anglicismes et leur censure systématique, réaction ulcérée devant l'anglomanie de l'Hexagone, saisie au vol du concept de $\alpha$ francophonie " dont la France n'a plus ni le privilège ni le contróle.

La langue et le nombril est entré en lice pour le Prix du Gouverneur général en décembre 1998 (catégorie essai). De nombreux articles parus dans la presse montréalaise, tous largement favorables, lui ont été consacrés : " La langue de chez nous " de Pascale Navarro, dans Voir $\mathrm{n}^{\circ} 13$, du 2 avril 1998, p. 41, s'interroge sur le * Québécois maniaque de la langue *. "D'une langue en crise au droit à la différence " de Raymond Giroux, dans Le Soleil du 16 mars 1998, p. B6, félicite Chantal Bouchard « d'avoir jeté la lumière sur la partie la plus dramatique de l'histoire québécoise, la grande noirceur duplessiste » et de s'être attardée sur la * crise identitaire ". A titre comparatif, * le Québec se fait-il tirer la langue? „ d'Odile Tremblay, dans Le Devoir du 20 octobre 1998, section Horizons, p. B1, fait le point sur les plus récentes publications sur la langue au Québec, fustigeant au passage Georges Dor pour son manque d'objectivité et son absence de formation académique. Justement, à ce 
propos, parce qu'elle résume la position de Chantal Bouchard, deux phrases citées dans cet article ont suscité notre intérêt. L'une, de Claude Poirier, directeur du dictionnaire historique de français québécois, préconise que l'on * [cesse] de reprocher aux gens de mal parler * et de tenir compte davantage de l'évolution globale de la langue, qui elle est positive. L'autre est de Jean Forest, qui dit en substance le mot de la fin : " il est inutile de se battre pour quelque chose qui s'améliore de façon spontanée ".

* Georges Dor, Anna braillé ène shot (Elle a beaucoup pleuré). Essai sur le langage parlé des Québécois, Montréal, Lanctôt éditeur, 1996, 192 p.; Louis Comelier, Plaidoyer pour l'idéologie tabarnaco, Montréal, BalzacLe Griot éditeur, 1997, 95 p.; Jacques Godbout, Richard Martineau, Le buffet, dialogue sur le Québec à l'an 2000, Montréal, Boréal, 1998, 120 p.; Marty Laforest, États d'âme, états de langues. Essai sur le français parlé au Québec, Montréal, Nuit blanche éditeur, 1997.

Houria Daoud-Brikci Université McGill 